\title{
The ACS Virgo Cluster Survey
}

\author{
Patrick Côté \\ Rutgers University, 136 Frelinghuysen Road, Piscataway, NJ 08854
}

Michael J. West

University of Hawaii, Hilo, HI 96720

\begin{abstract}
We describe the ACS Virgo Cluster Survey, an HST imaging survey of early-type galaxies in the Virgo Cluster. Multi-color ACS images for one hundred member galaxies, spanning a range of 450 in luminosity, are being used to study the central regions of these galaxies, their globular cluster systems, and the three-dimensional structure of Virgo itself. In terms of depth, spatial resolution, sample size and homogeneity, this represents the most comprehensive imaging survey to date of early-type galaxies in dense environments.
\end{abstract}

\section{Introduction}

The Virgo Cluster is the largest and most massive concentration of galaxies in the Local Supercluster. Not surprisingly, it has played a central role in understanding how galaxies form and evolve in dense environments, providing invaluable information on the extra-galactic distance scale, the nature of galactic nuclei, the local velocity field, and the shape and universality of the galactic luminosity function. In the study of early-type galaxies, Virgo has played an especially pivotal role since it contains, by far, the largest concentration of elliptical and lenticular galaxies in the local universe.

\section{Survey Strategy and Sample Selection}

With our colleagues (see below), we are carrying out an imaging survey of earlytype galaxies in the Virgo Cluster using ACS Wide Field Camera. While the full list of science drivers is too lengthy to describe in detail here, our primary scientific objectives include: (1) the measurement of distances for the full sample of galaxies using the method of surface brightness fluctuations; (2) a comprehensive isophotal analysis for each program galaxy, including the measurement of nuclear surface brightness and color profiles; and (3) the measurement of luminosities, colors, concentration indices, half-light radii, and ages for thousands of globular clusters belonging to our program galaxies.

The sample consists of one hundred early-type (E, E/S0, S0, dE, dE, N, and dS0) galaxies, drawn from the Virgo Cluster Catalog of Binggeli et al. (1985). Each galaxy is a confirmed member of Virgo based on its measured radial velocity. The program galaxies span the range $9.31 \leq B_{T} \leq 15.97$, corresponding to a factor of $\sim 450$ in luminosity. 


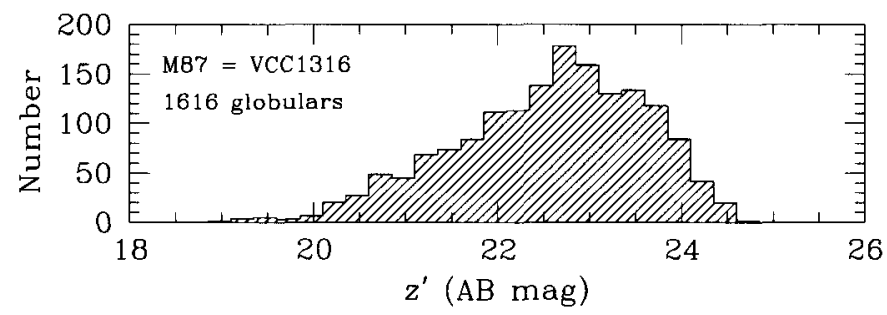

Figure 1. Globular cluster luminosity function for M87 from the ACS Virgo Cluster Survey.

The first data were obtained in December 2002, and the final images are expected to be in hand by April-June 2004. The dataset for each galaxy consists of two $375 \mathrm{sec}$ exposures in the $\mathrm{F} 475 \mathrm{~W}\left(\mathrm{~g}^{\prime}\right)$ bandpass, two 560 sec exposures in the F850LP $\left(\mathrm{z}^{\prime}\right)$ bandpass, and a single 90 sec F850LP exposure. This filter combination provides roughly a $50 \%$ increase in color baseline relative to $\mathrm{F} 555 \mathrm{~W}$ and $\mathrm{F} 814 \mathrm{~W}$, a commonly used WFPC2 filter combination. The approximate limiting magnitude in both band passes is $V \sim 25.7$ for a point-source signal-tonoise ratio of five. By observing each galaxy for just a single HST orbit, we are therefore able to sample the brightest $\sim 90 \%$ of the globular cluster luminosity function in each galaxy (see Fig 1).

\section{Reductions and Data Products}

A PYTHON-based calibration/reduction pipeline has been written to: align and combine the images; generate "background" images using multi-resolution wavelet filtering; detect objects; and measure object magnitudes and colors. Note that globular clusters at the distance of Virgo are marginally resolved on our images (WFC scale $=\simeq 3.8 \mathrm{pc} \mathrm{pixel}^{-1}$ ). For the globular clusters belonging to our program galaxies - identified on the basis of their colors and luminosities - we also derive structural parameters (i.e., half-light radii, concentration indices) by fitting psf-convolved, Michie-King models to their two-dimensional profiles. A complete discussion of the pipeline will be presented in Jordán et al. (2004), and additional information about the survey in general may be found at http://www.physics.rutgers.edu/ pcote/acs/

Acknowledgments. The ACS Virgo Cluster Survey is a collaborative effort between P. Côté, J.P. Blakeslee, L. Ferrarese, A. Jordán, S. Mei, D. Merritt, M. Milosavljević, E. Peng, J. Tonry, and M. West.

\section{References}

Binggeli, B., Sandage, A., \& Tammann, G.A. 1985, AJ, 90, 1681

Jordán, A., Côté, P., Blakeslee, J.P., Ferrarese, L., Mei, S., Merritt, D., Milosavljević, M., Peng, E., Tonry, J.L., \& West, M.J. 2003, ApJ, 613, 279 